\title{
The Efficacy of Wet Cupping on Blood Pressure among Hypertension Patients in Jeddah, Saudi Arabia: A Randomized Controlled Trial Pilot Study
}

\author{
Nouran Aleyeidi ${ }^{1},{ }^{*}$ Khaled Aseri ${ }^{2}$ and Abeer Kawthar ${ }^{3}$ \\ ${ }^{1}$ Community Medicine, Specialist Physician, Saudi Arabia \\ ${ }^{2}$ Community Medicine, Consultant Physician, Saudi Arabia \\ 3 Internal Medicine, Consultant Physician, Saudi Arabia
}

\begin{abstract}
Cupping is an ancient remedy that is known in many parts of the world. Wet cupping in specific is more popular in the Arab region, as it was a treatment recommended in Islam. Despite of that, it still needs more research to prove its efficacy on many diseases, and hypertension is one of them. The objectives of this study are to determine the efficacy of wet cupping on high blood pressure among hypertension patients. Also, to assess the incidence of wet cupping side effects in the intervention group. This pilot study is a two-armed randomised controlled trial with 10 participants assigned to the intervention group and 8 to the control group. The intervention group were high blood pressure patients who performed 3 sessions of wet cupping in addition to their usual anti-hypertension management, and the control group had high blood pressure patients who received their usual anti-hypertension management only. The results after 4 weeks of follow up showed no significant difference between the intervention and the control group in both the mean difference of systolic and diastolic blood pressure. There were no serious side effects of wet cupping in this study. In conclusion, wet cupping therapy was found to be efficient in reducing blood pressure in similar studies that was carried out before. However, this finding was not achieved in the present study due to the possibility of the small sample size, since this is a pilot study. More research is still recommended in that area.
\end{abstract}

Keywords: Hypertension; High blood pressure; Complementary method; Treatment; Therapy; Cupping therapy; Wet cupping; Hijama; Prophetic medicine; Saudi arabia

Abbreviations: HTN: Hypertension; WHO: World Health Organization; RCT: Randomized Controlled Trial; BP: Blood Pressure; SBP: Systolic Blood Pressure; DBP: Diastolic Blood Pressure; KAUH: King Abdulaziz University Hospital; SD: Standard Deviation; BMI: Body Mass Index; CVD: Cardiovascular diseases; 4W: Four Weeks; 8W: Eight Weeks; LDL: Low Density Lipoprotein; HDL: High Density Lipoprotein; TFT: Thyroid Function Test; LABE: Low Amount of Blood Extracted ; HABE: High Amount of Blood Extracted; STRICTA: Standards for Reporting Interventions in Clinical Trials of Acupuncture; CONSORT: Consolidated Standards of Reporting Trials

\section{Introduction}

Cupping therapy is an ancient remedy that has many ways of application. The two main types of cupping are dry cupping and wet cupping. In dry cupping, the skin is pulled into the cup by applying negative pressure. In wet cupping, they also form negative pressure on the skin but with lacerations on that area which allows the blood from the dermal microcirculation to be sucked into the cup [1]

There are mainly two methods of performing wet cupping. The first one is mainly used in Chinese Medicine and also in some German studies. It has five steps: demarcation (marking cupping points), sterilization, puncturing, cupping and sterilization. This method came to be known as (puncturing and cupping) or PC. The other method is well known in the Arab region and Islamic literature, and it has six steps: demarcation, sterilization, dry cupping of the desired site, puncturing, cupping and sterilization. This method has an extra cupping step before puncturing, so it may be called (cupping, puncturing and cupping) or CPC [2]. This is what The Arabic word "hijama" refers to, which was the highlight of this study.

Taiba Theory is the latest theory explaining the therapeutic effect of wet cupping or hijama. It states that the negative pressure applied by cups causes increase capillaries infiltration which results in local collection of filtered fluids, lymph and interstitial fluid in that area. This will dilute chemical substances, inflammatory mediators, and nociceptive substances, bathes nerve endings in collected fluids and breaks tissue adhesions. Following the puncturing of that area and by applying negative pressure once again, the fluids are evacuated and prevented from being reabsorbed by venous capillaries. This may increase immunity by skin wounding effect and boost release of endogenous opoids. Also, it enhances the blood circulation and lymph flow in that area in a short time period, which may help to regain homeostasis in the human body [3].

There are several systematic reviews that have been done to identify the efficacy of wet cupping on certain conditions. Some of those systematic reviews also assessed side effects of wet cupping that were mentioned in their primary studies. These studies stated that there was no serious adverse effect to cupping [4,5]. One larger systematic review was done to summarise all clinical research evidence of cupping therapy in China between 1959 and 2008. They identified 550 clinical studies, 73 of them were randomised controlled trials (RCTs), and there was no serious adverse effect reported in any of those studies [6]. The same author performed another systematic review to update his results and include 135 RCTs, 62 of them were new studies between 2008 and 2010, and there was still no serious adverse effect found in any of those trials [7].

A relevant systematic review was implemented which including all

*Corresponding author: Nouran A Aleyeidi, Community Medicine, Specialist Physician, Saudi Arabia, Tel: 00966504304577; E-mail: dr.nouran@live.com

Received December 11, 2014; Accepted January 20, 2015; Published February 10,2015

Citation: Aleyeidi N, Aseri K, Kawthar A (2015) The Efficacy of Wet Cupping on Blood Pressure among Hypertension Patients in Jeddah, Saudi Arabia: A Randomized Controlled Trial Pilot Study. Altern Integ Med 4: 183. doi:10.4172/23275162.1000183

Copyright: @ 2015 Aleyeidi N, et al. This is an open-access article distributed under the terms of the Creative Commons Attribution License, which permits unrestricted use, distribution, and reproduction in any medium, provided the original author and source are credited. 
the clinical trials that studied the efficacy of cupping on hypertension. The authors searched 15 databases without language restrictions and included all relevant trials until the end of June 2009. They found that only two studies met their criteria, one for dry cupping and the other for wet cupping. Both the studies showed some improvement in the blood pressure of the hypertensive participants, but their results were inconclusive. The authors of the systematic review concluded that further research is required in that area [8].

An important RCT, done by Zarei et al. assessing the efficacy of wet cupping in the treatment of hypertension was conducted in Iran and published in 2012. Its aim was to study the effect of wet cupping on blood pressure in 35-60 year old patients diagnosed with hypertension. In that study, 42 participants were divided randomly into 2 groups. The cupping group were prescribed a series of 3 staged wet cupping treatments at 2-week intervals in addition to their anti-hypertension medications, and the participants in the control group were only prescribed medication. The site for wet cupping was between the two scapulae, opposite the T1-T3 scapular spine. The final systolic and diastolic BP was measured after 6 weeks for comparison. The comparison of the means of systolic and diastolic blood pressures at the beginning and end of the study within the cupping group showed significant difference in the Systolic blood pressure (SBP) but not in the diastolic blood pressure (DBP). Also, the comparison of the means of the difference of systolic and diastolic blood pressure between the cupping and the control groups showed significant difference between groups by independent $t$-test for SBP, but also was not significant for DBP [9].

The objective of this study was to evaluate the efficacy of wet cupping therapy (Hijama) on the management of high blood pressure among hypertension patients. Also, to assess the incidence of wet cupping side effects among treated participants.

\section{Methods}

The study design was a randomized controlled trial pilot study design. It took place at King Abdulaziz University Hospital (KAUH) in Jeddah city in Saudi Arabia. The sample size was 18 participants, and they all performed wet cupping or hijama. A convenient sampling technique was used. The participants were included in the study after screening for the eligibility criteria. They were included if they had high $\mathrm{BP}$ at the time of the study (SBP $140 \mathrm{mmHg}$ or more and/or DBP 90 $\mathrm{mmHg}$ or more). For patients with DM, high BP is defined as SBP 130 $\mathrm{mmHg}$ or more and/or DBP $85 \mathrm{mmHg}$ or more [10]. They were aged between 19 and 65 years old. There were no gender restrictions. We excluded those with grade III Hypertension (SBP $180 \mathrm{mmHg}$ or more and/or DBP 110 BP or more). Also we excluded the patients with very high added risk, according to the WHO hypertension management guidelines [11]. Patients with secondary hypertension and pregnant women were also excluded. Also, if the patient performed dry cupping, wet cupping or acupuncture during the past 6 months then he or she was excluded from the study. After checking for eligibility, a written informed consent was taken from the participants and randomization was done. After randomization, 10 participants were assigned to the intervention group and 8 to the control group. Concealment was insured. Confidentiality of the participants was considered throughout the study. The participants were absolutely free to quit the study whenever they wished to do so.

The intervention under study was the performance of wet cupping or hijama. It took place at King Abdulaziz University Hospital, in the hijama clinic of the Scientific Chair of YA Jameel of the Prophetic
Medical Application. The hijama procedure is described as follows: Clean the areas with alcohol swab then put the cup on the area and start suction. Gently take of the cup and make 5 very superficial incisions parallel to each other. Replace the cup on the same area and repeat suctioning. Remove and replace the cups for about three times, then clean the area and do dressing. The hijama was carried out on four sites of the body. The first site is between the two scapulas, opposite the T1-T3 scapular spine (Al-Kahil), as done in Zarei's study [9]. The second site is located on the seventh cervical vertebra (GV14), as done in Guo's study [8]. The other 2 sites are on both sides of the neck. They are located 2 fingers posterior to the angle of the mandible on both sides, just below the skull bone, on the hair line (Al-Akhdaain). In the present study, three hijama sessions were conducted, once per month for 3 consecutive months. In Islamic literature, it is recommended to perform himaja on the $17^{\text {th }}, 19^{\text {th }}$ and $21^{\text {st }}$ days of the Arabic calendar months (lunar month) [12]. In this study we restricted from carrying out the hijama sessions on those specific days. The follow up period was 4 weeks after the final hijama session.

The main outcome of this study is BP measurements. All BP measurements were done on sitting position. A validated automatic oscillometric sphygmomanometer device (Omron 705IT) was used to minimise observer bias [13-15]. The BP measurement standards were followed as recommended by the Saudi Hypertension Management Guidelines [10]. The baseline BP measurement was recorded at the time of screening. If the participant was found to have high BP in the first screening time, then he or she will be scheduled for a second screening visit to repeat the BP measurement and confirm the diagnosis of high BP. Taking 2 BP measurements on 2 separate visits is the minimum acceptable by WHO and other more recent researches $[11,16]$.

The percentage of side effects that may appear as a consequence of the hijama procedure was calculated. Those side effects were monitored through data collection sheets that were filled by the interviewers immediately after each hijama session for immediate side effects, and after 4 weeks in the next visit for BP measurement to assess late side effects. The side effects were taken from the British Cupping society and from the article of clinical and histological changes after cupping therapy, published in 2012 [17].

Statistical analysis was done using SPSS program version 16.0. The baseline data were compared between the two comparison groups. A comparison took place between the SBP before hijama and the SBP after hijama by paired t-test. Also, the same was done to compare the diastolic BP before and after hijama. Following this, another comparison was carried on between the SBP and DBP differences a $\mathrm{P}$ value less than 0.05 was considered significant. Mean BP differences with $95 \%$ confidence intervals were reported. The per cent of any hijama side effect was calculated in the hijama group.

\section{Results}

There was no loss of follow up in this study. All the baseline variables were compared between the intervention and the control groups by Fisher's Exact or Student's t-test, and there was no significant difference in anyone of them between the two groups (Table 1). Most of the participants in this study were already taking anti-HTN medications, 8 from the intervention and 5 from the control group, and they continued taking them during the study.

After 4 weeks of follow up, the BP measurement was repeated for both groups. The baseline $\mathrm{BP}$ in the control group was compared to the $\mathrm{BP}$ reading after 4 weeks within each one of the groups by paired 


\begin{tabular}{|c|c|c|c|}
\hline Baseline characteristics & Intervention Group & Control Group & $P$ value \\
\hline $\begin{array}{c}\text { Mean Baseline Systolic Blood Pressure ( } \pm \\
\text { SD) }\end{array}$ & $160( \pm 13.5)$ & $149( \pm 11.1)$ & $0.090^{*}$ \\
\hline $\begin{array}{l}\text { Mean Baseline Diastolic Blood Pressure } \\
\qquad( \pm \text { SD })\end{array}$ & $91( \pm 10.3)$ & $94( \pm 10.6)$ & $0.566^{*}$ \\
\hline Mean age in years $( \pm S D)$ & $52( \pm 7.2)$ & $49( \pm 9.5)$ & $0.504^{*}$ \\
\hline Male:Female ratio & $4: 6$ & $3: 5$ & --- \\
\hline Diabetes (\%) & $4(40 \%)$ & $2(25 \%)$ & $0.638^{*}$ \\
\hline Hyperlipidemia (\%) & $6(60 \%)$ & $1(12.5 \%)$ & $0.066^{*}$ \\
\hline BMI $( \pm$ SD $)$ & $32.9( \pm 6.7)$ & $32.3( \pm 5.1)$ & $0.444^{*}$ \\
\hline Currently Smoking (\%) & $1(10 \%)$ & $1(12.5 \%)$ & $1.0^{*}$ \\
\hline Family history of premature CVD death (\%) & $0(0 \%)$ & $2(25 \%)$ & $0.183^{*}$ \\
\hline Mean Fasting Blood Sugar in $\mathrm{mmol} / \mathrm{L}( \pm \mathrm{SD})$ & $7.6( \pm 3.8)$ & $8.1( \pm 3.0)$ & $0.773^{*}$ \\
\hline Mean Cholesterol in $\mathrm{mmol} / \mathrm{L}( \pm \mathrm{SD})$ & $5.1( \pm 1.1)$ & $6.5( \pm 1.5)$ & $0.123^{*}$ \\
\hline Mean Triglyceride in $\mathrm{mmol} / \mathrm{L}( \pm \mathrm{SD})$ & $1.8( \pm 1.3)$ & $3.0( \pm 3.0)$ & $0.562^{*}$ \\
\hline Mean Creatinine in $\mathrm{mmol} / \mathrm{L}( \pm \mathrm{SD})$ & $71.8( \pm 36.3)$ & $76.8( \pm 13.6)$ & $0.776^{*}$ \\
\hline Mean Potassium in $\mathrm{mmol} / \mathrm{L}( \pm \mathrm{SD})$ & $3.9( \pm 0.2)$ & $4.1( \pm 0.3)$ & $0.109^{*}$ \\
\hline Mean TFT in ulU/L ( \pm SD) & $5.2( \pm 7.4)$ & $3.2( \pm 1.3)$ & $0.664^{*}$ \\
\hline
\end{tabular}

* Student's t-test; $\$$ Fisher's exact test

Table 1: Comparison of the participants' characteristics before intervention

\begin{tabular}{|c|c|c|c|}
\hline & & Systolic BP & Diastolic BP \\
\hline \multirow{3}{*}{ Hijama Group ( \pm SD) } & At Baseline & $160( \pm 13.5)$ & $91( \pm 10.3)$ \\
\hline & After 4 Weeks & $158( \pm 15.9)$ & $91( \pm 9.3)$ \\
\hline & BP Difference* & $1.8( \pm 11.7)$ & $0.0( \pm 6.5)$ \\
\hline \multirow{3}{*}{ Control Group ( \pm SD) } & At Baseline & $149( \pm 11.1)$ & $94( \pm 10.6)$ \\
\hline & After 4 Weeks & $139( \pm 12.8)$ & $88( \pm 11.0)$ \\
\hline & BP Difference* & $10.5( \pm 19.1)$ & $5.4( \pm 11.6)$ \\
\hline$P$ value ${ }^{* *}$ (Confidence Interval) & & $0.250(-24.1$ to 6.7$)$ & $0.269(-15.6$ to 4.8$)$ \\
\hline
\end{tabular}

${ }^{\star} \mathrm{BP}$ at baseline - BP after 4 weeks; " Comparing the BP differences of the intervention and control groups by independent t-test

Table 2: Comparison of BP between the two groups after 4 weeks of follow up.

t-test. There was no significant difference for SBP or DBP within the intervention and control groups. The mean difference between the baseline BP reading and the BP reading after 4 weeks was calculated for each group and used for comparison, because the baseline SBP was lower in the control group (by $10.7 \mathrm{mmHg}$ ) even before intervention. Then, a comparison took place between the mean differences of BP in both the intervention and control groups by unpaired t-test. There were no significant differences of the mean difference of SBP or the mean difference of DBP between the intervention and control groups ( $\mathrm{p}$ value $=0.250$ and 0.269 respectively) (Table 2$)$.

There were no serious side effects of wet cupping in the hijama group. Only 4 of the 10 participants in the hijama group complained of a side effect after the treatment. They were all self-limited and lasted not more than 24 hours. However, the only side effect that lasted for about a week was the bullae formation at one site of hijama that one participant suffered from. It was found that this effect accrued due to the prolonged application of the hijama cup during suction more than the time recommended in the study methods. Fortunately, the area was completely healed after 1 week, with daily dressing and application of "Mebo" cream, although the patient had DM. The other side effects were milder but more common, for example headache was the commonest side effect in this pilot study. It affected 3 of the participants in the hijama group. It evoked immediately after hijama and lasted for no more than 2 hours after hijama. For more details about hijama side effects that appeared in this study (Table 3).

\section{Discussion}

The results of this study showed no significant difference in the
SBP or DBP between the hijama and control groups after 4 weeks of following up. We performed the hijama sessions on random days and did not stick to a specific timing of the lunar month. These results were not consistent with the previous similar studies. In the Zarei et al RCT study, there was a significant difference in SBP when compared with the control group after 6 weeks of follow up [9]. This might be due to chance since the sample size was too small in this pilot study.

The hijama was a safe procedure with no serious side effects. It was well tolerated by the participants as well. There were a few mild selflimited side effects. The most common side effect was dizziness followed by headache, affecting 3 and 2 participants out of 10 respectively. One participant suffered from itching at the site of hijama and another one was observed with bullae formation. All these side effects are considered mild if we compared them to any anti-HTN medication's side effects [11].

This study is one of the few studies covering this rare topic of wet cupping and hypertension. This study results are not for generalizability because of the small sample size. Despite of that, future studies in the same topic should consider applying some modifications in the method of wet cupping we used in this study. For example, using shorter intervals between the wet cupping sessions or the number of incisions in each hijama site. The sites of hijama were the same as used in the previous studies [8].

Although the sample size was small, but this study gave us a better understanding of the hijama procedure and its possible side effects. On the other hand, lack of blindness in this study, and in all previous wet cupping studies, is still an issue. There is no sham wet cupping available 
Citation: Aleyeidi N, Aseri K, Kawthar A (2015) The Efficacy of Wet Cupping on Blood Pressure among Hypertension Patients in Jeddah, Saudi Arabia: A Randomized Controlled Trial Pilot Study. Altern Integ Med 4: 183. doi:10.4172/2327-5162.1000183

\begin{tabular}{|c|c|c|}
\hline The adverse effect & Number of events & $\begin{array}{c}\text { Number of participants } \\
\text { who experienced this } \\
\text { adverse effect }\end{array}$ \\
\hline Dizziness & 4 & $3 / 10$ \\
\hline Headache & 2 & $2 / 10$ \\
\hline Bullae Formation & 1 & $1 / 10$ \\
\hline $\begin{array}{c}\text { Itching at the site of } \\
\text { hijama }\end{array}$ & 1 & $1 / 10$ \\
\hline
\end{tabular}

Table 3: The frequency of adverse effects that occurred after Hijama. The total number of participants who experienced side effects was $4 / 10$ participants.

until today, although there is sham dry cupping [18]. We recommend the development of a new sham wet cupping procedure to aid in any wet cupping intervention study. Of course, further research is still needed to study the efficacy of wet cupping in treating hypertension with a larger sample size and a longer follow up period.

\section{Acknowledgments}

This study was funded by King Abdulaziz University and the Scientific Chair of YA Jameel of the Prophetic Medical Application, in Jeddah city in Saudi Arabia.

Thanks to all the people who helped us in this study, Dr. Samiha Murad, Prof. Soad Jaouni, Dr. Rajaa Alraddadi, Prof. Magda Hagras. We would also like to thank those who helped in in data collection, Dr. Sumayya Kubeisy, Dr. Rothaina Saeedi, Dr. Waleed Bukhari, Dr. Saad Alhatemy and Dr. Mohammed Ghonaim. Thanks to Dr. Adil Ibrahim and Prof. Ahmed Mandil for their opinion and advice. Finally, we thank all the patients who participated in this study.

\section{ClinicalTrials.gov identifier: NCT01853371}

\section{References}

1. Lee MS, Kim JI, Ernst E (2011) Is cupping an effective treatment? An overview of systematic reviews. J Acupunct Meridian Stud 4: 1-4.

2. Mahmoud HS ESS (2013) Methods of Wet Cupping Therapy (Al-Hijamah): In Light of Modern Medicine and Prophetic Medicine. Alternative \& Integrative Medicine 02.

3. Mahmoud HS ESS (2013) Medical and Scientific Bases of Wet Cupping Therapy (Al-hijamah): in Light of Modern Medicine and Prophetic Medicine. Alternative \& Integrative Medicine 02.

4. Cao H, Zhu C, Liu J (2010) Wet cupping therapy for treatment of herpes zoster: a systematic review of randomized controlled trials. Altern Ther Health Med
16: 48-54.

5. Cao H, Liu J, Lewith GT (2010) Traditional Chinese Medicine for treatment of fibromyalgia: a systematic review of randomized controlled trials. J Altern Complement Med 16: 397-409.

6. Cao H, Han M, Li X, Dong S, Shang Y, et al. (2010) Clinical research evidence of cupping therapy in China: a systematic literature review. BMC Complement Altern Med 10: 70.

7. Cao H, Li X, Liu J (2012) An updated review of the efficacy of cupping therapy PLoS One 7: 31793

8. Lee MS, Choi TY, Shin BC, Kim JI, Nam SS (2010) Cupping for hypertension a systematic review. Clin Exp Hypertens 32: 423-425.

9. Zarei M, Hejazi S, Javadi SA, Farahani H (2012) The Efficacy of Wet Cupping in the Treatment of Hypertension. ARYA Atherosclerosis Journal 8: 1-4.

10. Asiri Y, Abassam T, Bawazir S, Bsata W (2011) Saudi Hypertention Management Guidlines Synopsis 3rd ed. Riyadh: The Saudi Hypertension Management Society.

11. Khatib OMN, El Guindy MS (2005) World Health Organization. Regional Office for the Eastern Mediterranean. Clinical guidelines for the management of hypertension. Cairo: WHO Regional Office for the Eastern Mediterranean.

12. El-Wakil A (2011) Observations of the popularity and religious significance of blood-cupping as an Islamic medicine. Contemporary Islamic Studies 2.

13. Wan Y, Heneghan C, Stevens R, McManus RJ, Ward A, et al. (2010) Determining which automatic digital blood pressure device performs adequately: a systematic review. J Hum Hypertens 24: 431-438.

14. Coleman A, Freeman P, Steel S, Shennan A (2006) Validation of the Omron 705IT oscillometric blood pressure monitoring device according to the British Hypertension Society protocol. Blood Press Monit 11: 27-32.

15. E Assaad MA, Topouchian JA, Asmar RG (2003) Evaluation of two devices for self-measurement of blood pressure according to the international protocol: the Omron M5-I and the Omron 705IT. Blood Press Monit 8: 127-133.

16. Katakam R, Brukamp K, Townsend RR (2008) What is the proper workup of a patient with hypertension? Cleve Clin J Med 75: 663-672.

17. Qasim Ali Al-Rubaye K (2012) The Clinical and Histological Skin Changes After the Cupping Therapy (Al-Hijamah). Journal of the Turkish Academy of Dermatology 6.

18. Lee MS, Kim JI, Kong JC, Lee DH, Shin BC (2010) Developing and validating a sham cupping device. Acupunct Med 28: 200-204. 